\title{
Online checking of radiation dose for Computed Tomography imaging investigations performed on the human patients
}

\author{
Alin CORDOS ${ }^{1}$ \\ ${ }^{1}$ Technical University of ClujNapoca \\ Cluj-Napoca, Memorandumului St., No. 28, 400114, Romania; \\ tel. +4 0264401 200, 401248, tel./fax +4 0264592055
}

Keywords: x-ray, radiation dose, $\mathrm{HL7}$, web, CT, dosimetry

\begin{abstract}
This article presents an online checking method to get the accumulated $\mathrm{x}$-ray radiation dose for Computed Tomography imaging investigations performed to the human patients. When the patient arrived to imaging center to request a new investigation the system will generate an online verification based on the CNP. The software application will show a warning message if the radiation doze is exceeded. The software technologies used are based on web services and HL7 Version 2.3.1 standard.
\end{abstract}

\section{INTRODUCTION}

\section{Radiations}

Radiations are a special motion of matter, a form of energy released at the atomic level. Depending on the mode of propagation and their properties, there are two kinds of radiation:

1. electromagnetic radiation (wave) - are for example X-rays, gamma rays ultraviolet radiation, light radiation, infrared radiation, microwaves, radio waves. They are distinguished by their length and wave frequency. If the length is shorter, the radiation energy (hardness) is higher. X-rays are electromagnetic waves with wavelengths measured in angstroms. An Angstrom [1] is the 10000 part of a micron, so it is equal to 1/10000000 of a millimeter. X-rays used for medical purposes wavelength range from 0.06 to 8 angstroms, which gives them a high penetration.

2. corpuscular radiation -are electrically charged particles directly ionizing, as alpha and beta rays of radium and radioactive corpus, electrons, mesons, protons, etc. [1]

\section{Dosimetry}

Dosimetry represents the radiation dose absorbed by living tissues and is a key element in the study of biological phenomena caused by radiation.

Because $\mathrm{X}$ rays produce biological effects at different levels or at different types depending of the amount of energy transferred in any living organism, is absolutely necessary to know accurately the dose absorbed by different individuals or tissues exposed to radiation. Dosimetric assessments are needed in radiology to asses a balance between risk they may produce radiological investigations and patient benefit obtained by these investigations.

For medical purposes it is of great interest knowing dose of the energy absorbed in tissue: the amount of energy absorbed depends on the quality and quantity of $\mathrm{X}$ photons incident.

Quantitative dosimetry has three distinct aspects: dose of radiation, absorbed dose, equivalent dose. Emission dose is the amount of radiation emitted and it is expressed as R (rem).

Absorbed dose corresponds to the amount of energy absorbed by the body exposed to radiation and is expressed in rad or, in intermational system (IS) in Gy (gray). One Gy is equal to 100 rads. [1] Equivalent dose represents the biological effects assessment, secondary to radiation energy and especially with the type of radiation ( $\mathrm{X}$ and gamma photons, elementary particles). It is obtain by multiplying the absorbed dose by a factor of quality, which takes account of this phenomenon. 
Equivalent dose is expressed in rem or after SI in Sv (sievert).In the United States, radiation dosimeters measure and display dose in rems rather than sieverts. One rem $=1$ centisievert $=1 / 100$ sievert. [1]

\section{Biological effects of ionizing radiation}

Ionizing radiation can be dangerous to humans. Just as the sun can burn skin, so ionizing radiation can cause damage to the body. In their way, ionizing radiation, releasing enough energy to remove one or more electrons from atoms in irradiated tissue, thus disturbing the chemical activity of normal living tissue. A common hypothesis is that X-ray interacts with water molecules and generates hydroxyl radicals witch cause strand breaks or base damage in DNA. X-rays can also ionize DNA directly. Damages induced by radiations are rapidly repaired by various systems within the cell. DNA double-strand breaks are less easily repaired, and sometimes repair with errors and these facts can lead to gene mutations and chromosomal translocations, linked to the induction of cancer. (2) After a certain degree of disorder of these chemical processes, living cells cannot regenerate naturally and they permanently remain disturbed or die (if DNA damage).

X-rays influence all biological tissues. It will suffer some changes depending on the dose of radiation absorbed and can go until cell death. Biological effects are based on ionization radiation property of X-rays. In small doses $\mathrm{X}$ rays have biostimulation action. The first changes occur in the cell nucleus and consists in DNA damage and nuclear fragmentation.

Biological changes are also dependent on the type of cell that has been irradiated. From this point of view, cells are radiosensitive or radioresistant . Cell sensitivity to radiation is even greater as:

- reproduction activity is higher

- kariokinetic period and evolution is longer

- morphology and function are less fixed. [3]

Even if they are applied locally, X-rays have a general action on the body. If the body receives different amounts of rays at different time intervals the effects are added together and constitute cumulative action of ionizing radiation. If there are multiple radiation episodes, the tissue partially recover between irradiation and to achieve the same biological effect is necessary to apply a higher total dose, as in the case of a single irradiation.

Determinant factors of biological effects are:

1. The Dose Response Curve - For any biologically harmful agent, it is useful to correlate the dosage administered with the response of damage produced, in order to establish acceptable levels of exposure.

2. Rate of Absorption - The rate at which the radiation is administered or absorbed is important because a considerable degree of recovery occurs from the radiation damage and a given dose will produce less effect if divided or prolonged than if it were given as a single exposure.

3. Area Exposed - The portion of the body irradiated is an important parameter because the larger the area exposed, in the same conditions, the greater global damage to the organism.

4. Variation in Species and Individual Sensitivity - There is a wide variation in the radio sensitivity of various species. Lethal doses for plants, for example, are much larger than those for mammals.

5. Variation in Cell Sensitivity - there is a wide variation in susceptibility to radiation damage exists among different types of cells and tissues at the same individual. Cells which are rapidly dividing are more sensitive than those which do not divide. Most sensitive are lymphocytes, followed by immature red blood cells. Cells of low sensitivity include muscle and nerve, which are highly differentiated and do not divide. [3]

\section{CT and Its Use}

CT has transformed much of medical imaging by providing good quality views of the organ or body region of interest. Unfortunately, CT involves larger radiation doses than the more common, conventional x-ray imaging procedures. In table 1 there are a few examples of radiation doses taken 
by the patient at CT in various regions. The number of these investigation has increased rapidly, in the hole world due to advances in CT technology that make it extremely user-friendly, for both the patient and the physician. [4]

Table 1. Radiation doses taken by the patient at CT in various regions [4]

\begin{tabular}{lr} 
Imagistic investigation & Radiation (mSv) \\
\hline Full-body CT scan & 10 \\
CT scan: heart & 16 \\
CT scan: abdomen \& pelvis & 15 \\
Natural radiation we're all exposed to, per year & 2 \\
CT scan: head & 2 \\
Spine X-ray & 1.5 \\
Mammogram breast x-ray & 0.4 \\
Chest X-ray & 0.1 \\
Dental x-ray & 0.005
\end{tabular}

CT is used for different purposes of imaging: diagnosis in symptomatic patients or screening of asymptomatic patients, in all kinds of patients' population (adult or pediatric). CT for diagnosis is the largest of these categories. Lately, the use of CT in pediatric diagnosis tends to increase because of decrease in the time needed to perform a scan (less than 1 second), so the moving artifacts during image acquisition are prevented. For screening purposing, CT is increasingly used on groups of population at risk (virtual colonoscopy, CT lung screening for smokers, CT cardiac screening, etc) [5]

\section{Typical Organ Doses}

CT scanning will generate a considerably larger organ doses, comparing by conventional radiography (for example, abdominal $\mathrm{CT}$ will produce a dose for stomach 50 times larger than a conventional abdominal radiography).

There are many factors that influence the radiation doses to particular organs from any given CT study. This factors depend on examination technique (number of scans, scanning time), on the patient (size of the patient), or on the device (the axial scan range, the scan pitch, the tube voltage, the specific design of the scanner) [6]

\section{Risks Associated with Low Doses of Radiation}

There are epidemiologic studies how offers direct evidences that organ doses corresponding to a common CT study (two or three scans), result in an increased risk of cancer. [5] Studied organ usually receives a radiation dose in the range of 15 millisieverts ( $\mathrm{mSv}$ ) (in an adult) to $30 \mathrm{mSv}$ (in a neonate). (7) The children are at greater risk than adults because they have more remaining years of life to develop a radiation-induced cancer and because they are inherently more radiosensitive. [5] It is a consensus in medical world that patients' exposure to radiation through medical imaging have to be reduced. This goal can be achieved by 3 general approaches:

1. CT techniques and examination protocols should be standardized and optimized to limit the radiation associated with individual scans.

2. Attempt to reduce the number of $\mathrm{CT}$ examinations will minimize medical radiation exposure. There is statistical data suggesting that up to $30 \%$ of the CT examinations currently performed may be unnecessary.

3. Tracking and collecting dose information at the patient level because patients may undergo repeated imaging over time. An electronic medical record could minimize individual radiation dose, especially among patients who have repeated imaging and who are at greatest risk of radiation-associated cancer. [8] 


\section{DATABASE STRUCTURE}

Storing data is made in a database that can be installed in a data center or a public cloud type structure. Server that manages the database is an MS SQL 2008 R2.

Data is stored in this database automatically or manually:

- Automatic storage is accomplished using a software aplication that extract information about radiation dose from DICOM files generated by CT device.

- Manual storage is realized through web applications that connect directly to the database. Through this web portal an operator can manually enter information on the amount of radiation to which each patient was exposed.

Each patient is uniquely identified in the database by CNP number.

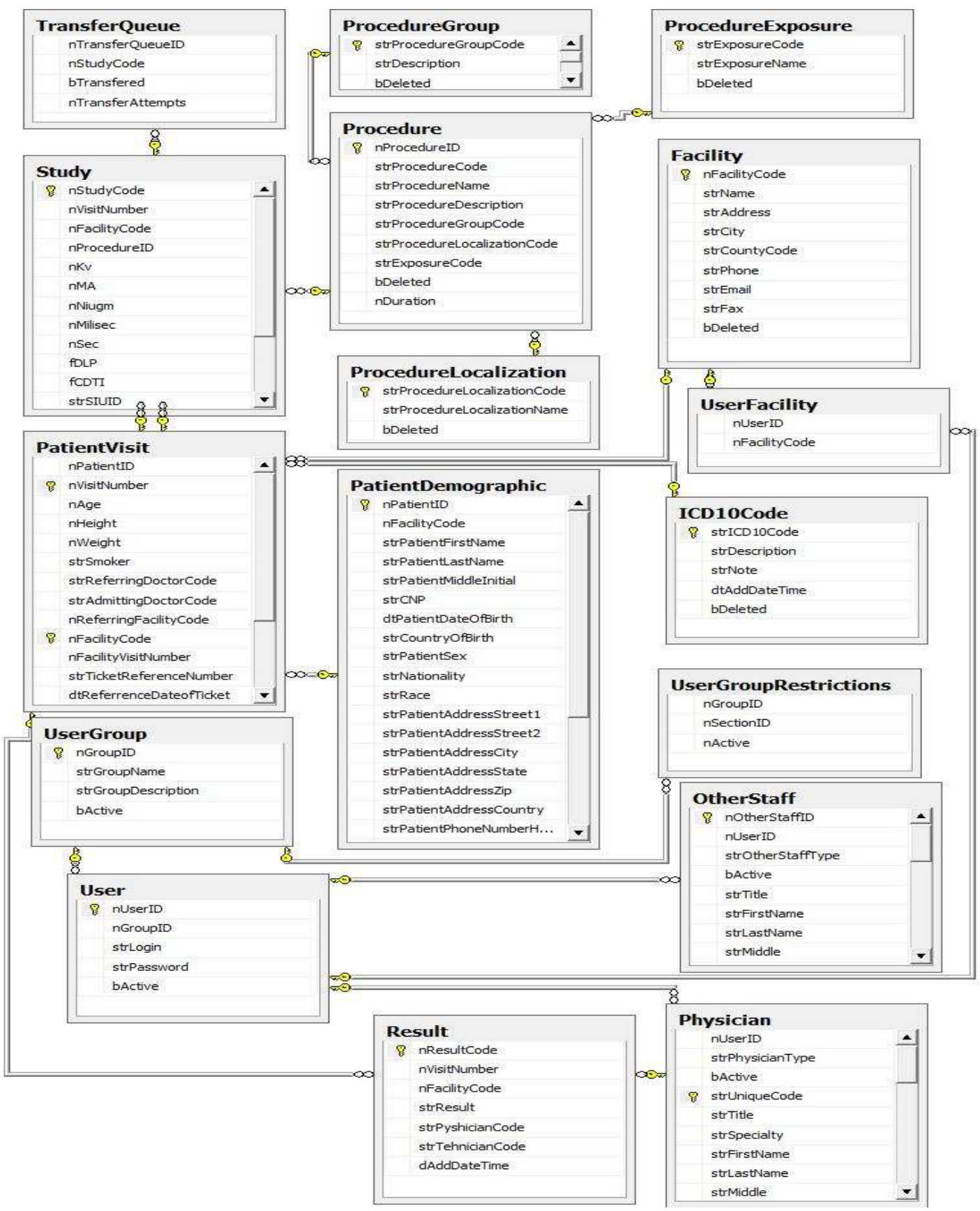

Fig. 1. Database structure 
The information regarding patients and their visits are stored in two tables called "PatientDemographic" and "PatientVisit". The relation between these tables are made by a simple data base key called "nPatientID". This key is a primary key in "PatientDemographic" table, it related only one patient. The data base relation between these two tables is 1:N. [9]

The information about dose value and information about studies are stored in the table called "Study". This table has a data base relation with "Procedure" table. Through this table we obtain information about procedures that was made during imaging examination. The data base relation between "Study" table and "Procedure" table is made by a simple key called "nProcedureID". This is primary key in "Procedure" table and foreign key in "Study" table.[9]

The data base relation between patient and his exams are made through "PatientVisit" table. The "Study" table has a data base relation 1:N with "PatientVisit" table. That means one visit can have more than one study. The relation between "Study" table and "PatientVisit" table is made by a multiple key, "nVisitNumber" and "nFacilityCode". This kind of relation allows to each facility (imaging center) to have its own visit number generator.[10]

The "Facility" table contains information about imaging center where the Computed Tomography device was installed. The facility code is unique on region or country.

The tables: "ProcedureGroup", Procedure Localization, ProcedureExposure and "ICD10Code" are tables where the software application store additional information about procedure and investigation that are made in each medical imaging center. [11]

The "User" table belongs to administrative component of data base structure. The software application stores in this table information about users who can access the data base information. The users are organized by groups of access and they are related to each facility. The database access rights are made by these groups of access (read, write, query, management reports, etc.). The connection between "User" table and "UserGroup" are made by a simple database key called "nGroupID", which primary key in "UserGroup" table and foreign key in "User" table. The database relation is $1: \mathrm{N}$, that means more than one user should belongs to the same access group of rights.[9]

The users are split in two tables: the physician table called "Physician" and "Otherstuff" table.

If the imaging centers wants to store the radiologic result for each visit, they can do it because the software application can store the result in "Result" table, which are connected to "PatientVisit" table through foreign key "nVisitNumber".[11]

The software application from all medical imaging centers was design to make an interconnection with a central database. This central database can be regional, national or can be a public cloud with special security access for specified users.

\section{DOSIMETRY SYSTEM - EXTERNAL ACCESS}

To avoid over exposure to X-rays of patients is required online verification of each patient when they are presented to an imaging center to demand a new Computed Tomography investigation. By checking this radiation dose the system will get radiation dose built up over a period of time, date of the last time-based X-ray imaging and anatomical part of the body that was investigated. Information about anatomical areas investigated and date of investigation done is very important to avoid scanning same anatomical areas in a short time. 


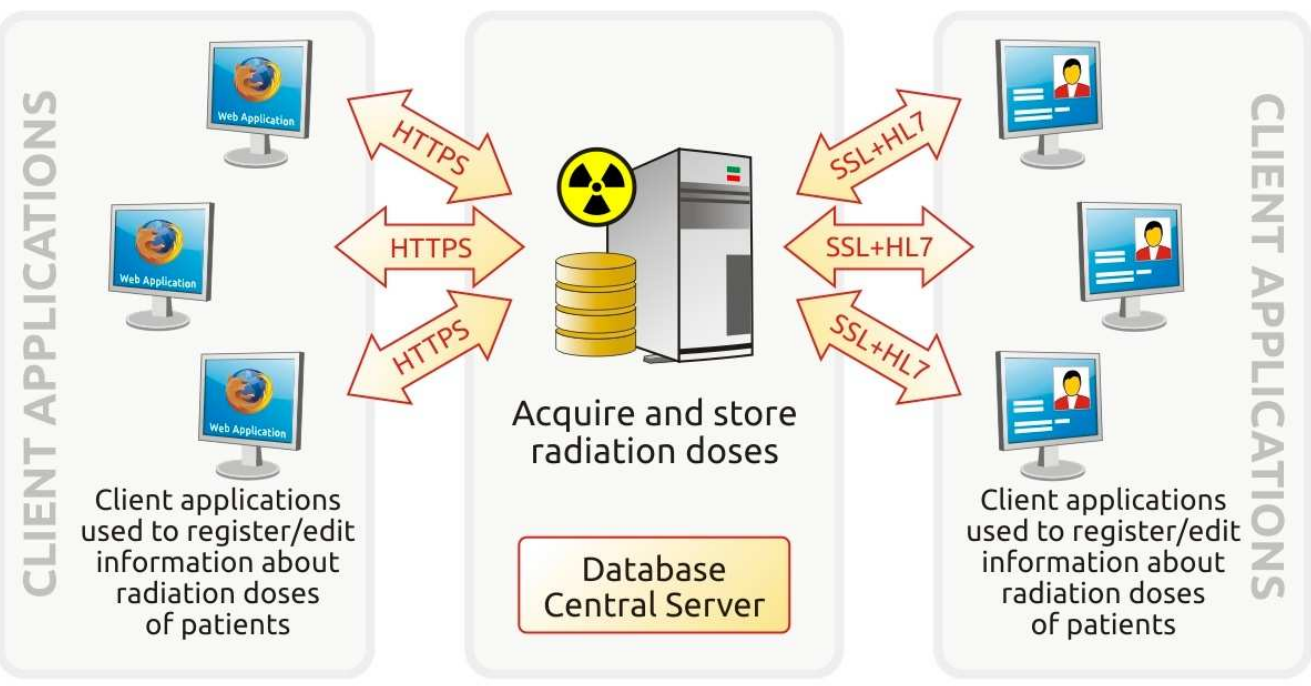

Fig. 2. Dosimetry system - communication interfaces

The dosimetry system provide two general ways to access information:

1. By using web services technologies and ASP/ASP.net;

2. By using the HL7 version 2.3.1.

Using these two methods any software application can get real-time information about each patient based CNP(patient unique ID). The dosimetry information can be stored at regional or national level.

\section{POST PROCESSING DATA}

Data stored in the data center or public cloud may be viewed either through web applications that can generate a wide range of statistical reports by each institution interest or can be a simple CNPbased verification, when we want to know the total amount of radiation accumulated for a given patient from imaging investigations conducted to date.

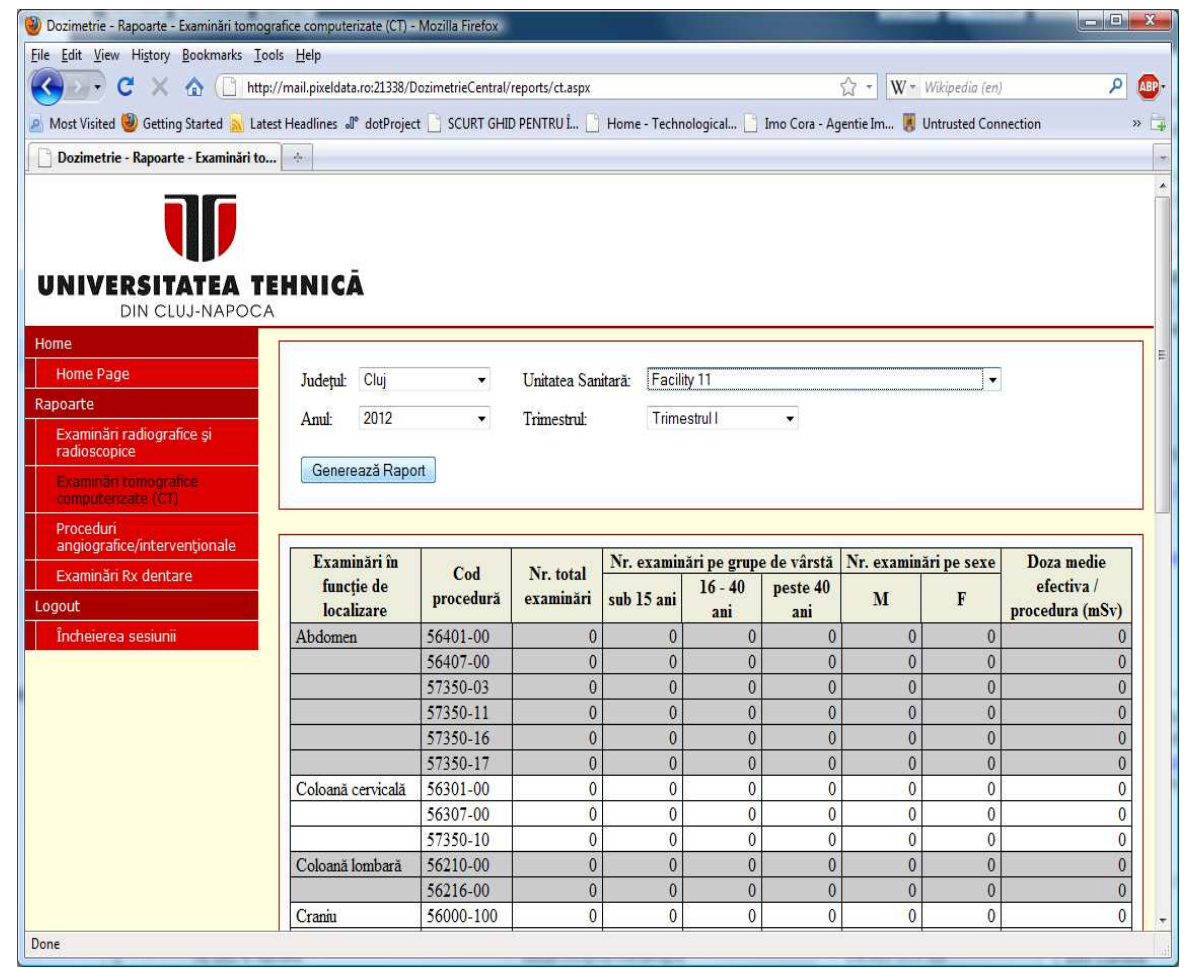

Fig. 3 Dosimetry report [5] 
There are two main purposes:

1. Statistical reports - in this moment is very useful to generate statistical statements on forms required by the Department of Public Health, or the Department of Radiation Hygiene

2. To obtain information - this option is used especially by software who wish to connect to the centralized database and obtain information about dosimetry based on CNP. The software application responsible for acquisition, centralization and storege information on patient radiation dose accumulated after performing CT imaging, provides to the software developers two different ways to access and query the system:

a. A first method is by using web services - access is done using C\#, ASP.net.

b. The second method is for medical applications using HL7 Version 2.3.1 standard. They can connect to the software application using query type HL7 message to obtain the desired information. To secure information can be used SSL protocol to encrypt information transmitted over 128-bit key.

\section{CONCLUSIONS}

This software application can provide a real-time information on the level of radiation to which the patient was exposed and alerts on situations contraindications for patients suffering from certain chronic diseases or pregnant women especially in the first three months of pregnancy when all Xray investigation method is an absolute contraindication.

The dosimetry reporting system is an "open system" because it implemented two special interfaces to get information from its data base. This feature is very important for medical software companies because they can perform a secure connection to dosimetry system and find out information about radiation dose for any patient.

The system was designed and developed to work in public or private cloud systems. Its external interfaces are web based or HL7 standard compliant. The external software applications can use both of these interfaces.

This software application can provide, for the first time, the ability to keep under control the complex processes taking place in a medical institution or center for diagnosis and treatment based on CT scanners.

\section{Appendix (Acknowledgments)}

This paper was supported by the project "Development and support of multidisciplinary postdoctoral programmes in major technical areas of national strategy of Research - Development Innovation" 4D-POSTDOC, contract no. POSDRU/89/1.5/S/52603, project co-funded by the European Social Fund through Sectoral Operational Programme Human Resources Development 2007-2013.

\section{REFERENCES}

[1] Alina Adriana Feiler, Ana-Maria Ungureanu, Manual de radiologie şi imagistică medicală, vol 1, Editura Victor Babeş Timisoara, 2012

[2] Mitelman F, Johansson B, Mertens FE. Mitelman database of chromosome aberrations in cancer. Cancer Genome Anatomy Project, 2007. (Accessed November 5, 2007, at http://cgap.nci.nih.gov/Chromosomes/Mitelman.)

[3] UW Environmental Health and Safety Principles of Radiation Protection Section 2 Biological Effects of Ionizing Radiation, January 2006

[4] Martin CJ, Sutton DG, Sharp PF. Balancing patient dose and image quality. Appl Radiat Isot 1999;50:1-19 CrossRef | Web of Science | Medline 
[5] David J. Brenner, Ph.D., D.Sc., and Eric J. Hall, D.Phil., D.Sc., Computed Tomography An Increasing Source of Radiation Exposure, N Engl J Med 2007; 357:2277-2284November 29, 2007DOI: 10.1056/NEJMra072149

[6] Martin CJ, Sutton DG, Sharp PF. Balancing patient dose and image quality. Appl Radiat Isot 1999;50:1-19 CrossRef | Web of Science | Medline

[7] Brenner DJ, Doll R, Goodhead DT, et al. Cancer risks attributable to low doses of ionizing radiation: assessing what we really know. Proc Natl Acad Sci U S A 2003;100:13761-13766

[8] Rebecca Smith-Bindman, MD; Jafi Lipson, MD; Ralph Marcus, BA; Kwang-Pyo Kim, PhD; Mahadevappa Mahesh, MS, PhD; Robert Gould, ScD; Amy Berrington de González, DPhil; Diana L. Miglioretti, PhD, Radiation Dose Associated With Common Computed Tomography Examinations and the Associated Lifetime Attributable Risk of Cancer, Arch Intern Med. 2009;169(22):2078-2086. doi:10.1001/archinternmed.2009.427.

[9] Cordos Alin, Aurel VLAICU, Bogdan ORZA, Angela CORDOS, Automatic Acquisition Methods Of X-Ray Doses From Computed Tomography Modalities, QIEM, November 2012

[10] Cordos Alin, Acquisition And Processing Information Concerning The Röntgen (X-Ray) Radiation Dosage In Medical, Seminar IS 2011 / Technological Development in a Sustainable Economy, Apr.2011

[11] Cordos Alin, Acquisition Methods Of X-Ray Doses, Seminar TM 2012 / Technological Development in a Sustainable Economy, Mar.2012 